\title{
Ciencia subatómica de la palabra en Quânticos da Incerteza de André Carneiro*
}

\section{Resumen}

En el poemario Quânticos da Incerteza (2007), el escritor (pintor, fotógrafo y cineasta) André Carneiro pone en juego el universo conceptual de la mecánica cuántica (fundamentalmente el principio de incertidumbre de Werner Heisenberg) para apuntalar las inflexiones reflexivas en torno a los usos y estatuto de la palabra. Carneiro, quien fuese además el indiscutible decano de la ciencia ficción brasilera, reúne y articula en este texto las concepciones de dos órdenes simbólicos escindidos a partir del Siglo de las Luces: la ciencia y la poesía.

Palabras clave: André Carneiro; Quânticos da Incerteza; mecánica cuántica; palabra; poema.
Citar: Martínez, L. (julio-diciembre 2018). Ciencia subatómica de la palabra en Quânticos da Incerteza de André Carneiro La Palabra, (33), 33-43. doi: https://doi. org/10.19053/01218530.n33.2018.7639

\section{Luciana Martínez}

Doctora en Humanidades y Artes (mención en Literatura) por la Universidad Nacional de Rosario, Argentina. Docente en las cátedras de Literatura Europea II (Carrera de Grado en Letras) y Estética II (Carrera de Grado en Bellas Artes) en la misma casa de estudios. Es además Investigadora en el Instituto de Estudios Críticos en Humanidades (IECH, UNR-CONICET). lmartinez@iech-conicet.gob.ar luciananmartinez@gmail.com

*Artículo de reflexión enmarcado en el proyecto de investigación: «Ficcionalizaciones de la ciencia en formulaciones realistas: Severo Sarduy, André Carneiro, Mario Levrero y Marcelo Cohen». 


\section{la palabra}

\section{Subatomic Science of the Word in Quânticos da Incerteza [Uncertainty Quantics] by André Carneiro}

\section{Abstract}

In the poetry book Quânticos da Incerteza (2007) the writer (painter, photographer and film-maker) André Carneiro brings the conceptual universe of quantum mechanics into play (Heisenberg's indeterminacy principle, fundamentally) in order to point out reflective inflections on the uses and status of the word. In this text, Carneiro, the undeniable dean of Brazilian science fiction, brings together and articulates the conceptions of two symbolic orders that have been divided since the Age of Enlightenment: science and poetry.

Keywords: André Carneiro; Quânticos da Incerteza; quantum mechanics; word; poem

\section{Science subatomique des mots dans Quânticos da Incerteza [Quantiques de l'incertitude] d'André Carneiro}

\section{Résumé}

Dans le recueil de poèmes Quânticos da Incerteza (2007), l'écrivain (également peintre, photographe et cinéaste), André Carneiro utilise l'univers conceptuel de la mécanique quantique (et plus précisément le principe d'incertitude de Werner Heisenberg) pour mettre en avant une réflexion autour de l'utilisation et du statut des mots. Alors que depuis le siècle des Lumières, science et poésie étaient deux conceptions symboliques distinctes, Carneiro, précurseur de la science-fiction brésilienne, les réunit et les articule dans ce texte.

Mots-clés: André Carneiro; Quânticos da Incerteza; mécanique quantique; mots; poème. 


\section{André Carneiro en contexto}

Como una planta salvaje que crece forzando la apertura de las grietas entre el cemento, a mediados del siglo XX surge en Brasil un movimiento literario cuyas condiciones de posibilidad paradójicamente no existían en el terreno de la realidad material. Contra todo pronóstico, durante la década del 50 emerge en el terruño lusófono, de forma contemporánea al movimiento del tropicalismo que sería su marca cultural distintiva, un grupo de autores abocados al cultivo de un género que, por aquel entonces, comenzaba a expandirse mundialmente desde EE.UU., lugar que fue reconocido como su centro neurálgico: la "ciencia ficción". ${ }^{1} \mathrm{Se}$ trató, en definitiva, del primer corpus coherente y original de ficción científica brasilera (Causo, 2003, p. 289).

Ante la carencia de un contexto científico tecnológico que alimentara la usina de ideas literarias en su realidad local inmediata (como, por el contrario, sucedió en EE. UU. o posteriormente en Japón), la ciencia ficción brasilera encontró, no obstante, su necesario motor en otras literaturas traducidas, principalmente en la traducción de las obras de Julio Verne y $\mathrm{H}$. G. Wells y, posteriormente, en la de figuras clásicas del género, como Asimov, Bradbury, Heinlein, entre otros (Pereira Gonçalves Camara, 2005). De forma similar a Pablo Capanna (con quien Carneiro compartió espacios de publicación en las revistas de ciencia ficción rioplatenses durante la década del 80) describe el posterior desarrollo del género en Argentina, la "ciencia ficción" brasilera se moduló a sí misma como un ejercicio imaginativo movilizado por la lectura de Otras literaturas, lo que propició que esta se pusiera en sintonía con cierta sensibilidad distópica que comenzaba a recorrer el géne- ro a partir de la Guerra Fría, al tiempo que se impregnaba y hacía eco de una retórica optimista que atravesaba en el terreno local a la nación entera y sus expectativas de desarrollo. La "generación GDR" (en honor a la editorial que dio lugar a las primeras antologías de autores locales) surgió en el contexto del boom editorial conciliando desde sus orígenes aspectos antagónicos: la distopía y el optimismo conformaban la sensibilidad de una manifestación literaria cuya existencia material no se encontraba habilitada por ninguna tradición protogenérica que la precediera, ni tampoco por una figura aglutinante que oficiara de cabeza editora o delimitara criterios estéticos de grupo o movimiento. ${ }^{2}$

Ahora bien, a pesar de su no concertada homogeneidad, esta "Primera onda" de la ciencia ficción (como también se la llamó), contó con ciertas figuras cuya centralidad terminó por

\footnotetext{
Existe, en este como en tantos otros sentidos con respecto a esta manifestación literaria, una controversia medular. Sam Lundwall, uno de los más grandes ensayistas y editores de género, argumenta con notable lucidez y solvencia que la primera y más importante vertiente de la ciencia ficción es europea, la cual explora libremente temas de la filosofía y la metafísica conjuntamente con hipótesis propias del campo científico. Esta línea de la ciencia ficción (cuyos más grandes precursores serían H. G. Wells, Olaf Stapledon y C. S. Lewis) habría sembrado el camino para el rebrote del paradigma de la mística que es distintivo de las manifestaciones del género más actuales, como así también sería antecedente de todas aquellas expresiones latinoamericanas que no terminan de ser consideradas parte de la ciencia ficción precisamente por la imagen del género que se construyó desde EE. UU. a partir de la conocida Edad de Oro campbelliana. En este sentido, el modelo de tratamiento riguroso de los temas científicos que se impuso a partir del avance de las publicaciones estadounidenses (y su consecuente concepto de Science-Fiction) obturó que se reconocieran otras vertientes de la "ciencia ficción", como las que se cultivaban en Europa o más tarde en Latinoamérica (Lundwall, 1986).

2 A diferencia de Argentina, que contaba con los antecedentes de la "fantasía científica" (Gasparini, 2012) de figuras como Eduardo Ladislao Holmberg, Leopoldo Lugones, Horacio Quiroga (entre otros), Brasil no disponía del mismo caldo de cultivo. En tierra lusófona, esto sucede casi sin excepción, descontando el evidente interés de una figura notable como Machado de Assis por la psiquiatría y las "ciencias de la mente" ("O alienista", 1882; "Conto Alexandrino", 1884; entre otros) y de algunos textos aislados de Monteiro Lobato (fundamentalmente o presidente negro, 1926).

Por lo demás, como expresarían Andrea Bell y Yolanda Molina Gavillán, "There was no cohesive science fiction tradition in Spanish and Portuguese-speaking world, and no Gernsback or Campbell to nurture writers and give the emerging genre a distinct shape and feel" (2003, p. 4).
} 
imponer una organización a su constelación. Dentro de ella, el paulista André Carneiro ocupó, sin duda, un lugar especial; no solo por haber captado a partir de la década del 60 la atención de críticos y escritores de ciencia ficción a nivel internacional (prueba de ello es su destacada consideración en la que fuera la primera tesis escrita sobre la ciencia ficción brasilera, del norteamericano David Dunbar) $\mathrm{y}$ haber sido publicado en diversas antologías junto con figuras de consagración, sino por su sostenida producción ficcional y crítica dentro del género. ${ }^{3} \mathrm{Si}$ una figura como Pablo Capanna tiene en Argentina el mérito de haber escrito la primera historia de la ciencia ficción en español (El sentido de la ciencia ficción, 1967), a André Carneiro debe adjudicársele, simultáneamente, la primera reflexión de largo aliento sobre el tema en lengua lusófona: Introdução ao Estudo da 'Science Fiction' (1967).

La evidente veta crítico-ensayística de Carneiro viene a complementarse, además, con su interés por la actividad de divulgación, moneda frecuente entre los escritores del género, que siempre suelen apelar a ciertos acervos conceptuales para erigir luego el universo de sus ficciones. En este senti- do, Carneiro comparte un interés por la parapsicología y las teorías sobre la hipnosis, que lo aproxima asimismo a otros escritores cercanos al género, como por ejemplo al uruguayo Mario Levrero (con quien además intercambió correspondencia). La escritura de sus manuales de divulgación científica sobre dichos temas, vuelve visible una inquietud marcada por la ciencia que, no obstante, siempre se conecta con la actividad artística o, mejor dicho, con el cultivo de las artes en plural. Y digo esto, porque a pocos hombres podría aplicárseles con tanta facilidad el calificativo de «polifacético» como a André Carneiro.

En el campo de las artes, Carneiro cultivó la poesía, la narrativa (cuento y novela), la producción audiovisual, la fotografía, la pintura. Pero si hablo de «hombre» polifacético, y no meramente de «artista», es porque su espíritu creativo no se restringió a la exploración de las múltiples expresiones del arte, sino que lo orientó a realizar un esfuerzo genuino por conciliar las «dos culturas» (Snow, 2000), es decir, aquellos dos órdenes simbólicos escindidos históricamente a partir del Siglo de las Luces; estos son: el arte y la ciencia. En este ca- mino, la estética no puede sino aspirar a lo gnoseológico, y lo gnoseológico tomar forma estética. Hombre de múltiples artes, entonces, y conocedor profundo de los modelos científicos, Carneiro no solo se interiorizó a lo largo de su vida con distintas teorías consagradas dentro del campo epistemológico, que lógicamente encontraron un destino final en la fagocitación ficcional, sino que llevó a cabo una fuerte tarea de divulgación de la parapsicología, disciplina de la que funcionó como principal importador en Brasil.

Aquel sueño romántico que se expresa con prístina claridad en el Fragmento 115 («todo arte debe hacerse ciencia y toda ciencia, arte»), expresión de un anhelo totalizante que surge como síntoma a contramano del inevitable proceso de compartimentación que produjo la lógica del racionalismo imperante durante el período de consolidación de la ciencia moderna, es plasmado por Carneiro en el que fuese tal vez uno de los terrenos más fértiles para pensar las relaciones entre literatura y ciencia. Me refiero a aquél género que de alguna manera desafía el imperativo moderno de escisión disciplinar: la ciencia-ficción.

3 Sorteando los estigmas que han recaído históricamente sobre el género desde el punto de vista académico, es elocuente en este sentido que hacia 1976 se haya realizado bajo la pluma del por aquel entonces estudiante de postgrado, David Dunbar, la primera tesis de doctorado sobre la ciencia ficción brasilera (Dunbar, 1976). Dunbar había realizado durante la década del 70 una extensa investigación de campo en Brasil, momento en el que tuvo oportunidad de entrevistar a André Carneiro, autor que su director de tesis, Leo Barrow, había traducido con motivo de su publicación en una antología del género realizada en EE. UU. 
Más allá de cómo esta inquietud holística se expresa en sus ficciones narrativas, aspecto que he tratado en trabajo anteriores (Martínez, 2018), lo cierto es que esta idea aparece explícitamente en sus propias reflexiones en torno al género. El binomio «ciencia-ficción» (heredado de una importación desatinada, extremadamente respetuosa, del concepto de Science-Fiction que se forjara en el circuito editorial estadounidense hacia mediados de la década del 20) reúne en sí la ciencia y la ficción en una palabra compuesta en la que ambos términos se ven mutua e inevitablemente afectados, nota Carneiro en su Introdução ao Estudo da 'Science Fiction' (1967, p. 12). En su asociación, la «ciencia» no es simplemente teoría sino ficcionalización de la teoría, y la «ficción» deja de ser mera ficción para pasar a ser posibilidad científica, especulación ontológica de algo que a priori no es.

En este contexto, la ficción narrativa es, al igual que la lírica, laboratorio de experimentación sobre las posibilidades de reunión de los elementos de este complejo binomio. Poco importa si la poesía de Carneiro puede o no entenderse como participando de este género (hay, en efecto, textos clásicos como el de Darko Suvin que permitirían realizar una lectura en esos términos, a riesgo de quedar sumidos en un debate taxativo sobre qué es ciencia ficción y qué no) en el que la relación entre literatura y ciencia alcanza un grado máximo y evidente de cristalización; lo crucial, más allá de la lectura genérica, radica justamente en visualizar la presencia de dicha relación jugándose en el seno de una especulación que, en este caso, se torna lírica.

En consecuencia, al igual que sucede con el devenir de la ciencia, la obra poética de Carneiro muestra una progresión histórica en su interés, la cual avanza en su indagación desde lo macro hacia lo micro. Si en su segundo poemario, Espaçopleno (1966), los protagonistas son los planetas, galaxias y constelaciones («o planetário»), submarinos («ar»), rayos x y microscopios que permiten estudiar bacilos («raio $\mathrm{x} »)$, pero también telescopios («corrida no espaço») y robots («ficção científica») que articulan imaginarios del fin del mundo en donde la entropía desintegra las ciudades y las fisonomías humanas que habitan el universo material percibido ( $\ll$ o dia final»), en un texto tardío como Quânticos da Incerteza (2007), el interés científico de sus poemas termina de virar hacia otros paradigmas que no son los de la ciencia moderna clásica, soberana del paisaje tecnológico que impregna el universo macroscópico al menos desde el siglo XIX. La voz y mirada líricas se desplazan de lo macro hacia lo micro, y en ese giro la ciencia se orienta hacia una especula- ción en torno a lo más seminal: la unidad mínima de la lengua como fundamento de todo lo que es.

Por eso, en este contexto, el propio estatuto del acto enunciativo (los usos y husos de la palabra) es pensado en el marco de las teorías que se presentan. En esta cosmovisión integral, que reúne arte y ciencia, Carneiro plantea otros interrogantes, desde otros paradigmas, pero también otras resoluciones. Palabra y ciencia conforman una indisociable amalgama en la que la ciencia vuela libremente con alas líricas y la palabra adquiere entidad y peso científicos; una poesía que, como el sueño romántico, se convierte en ciencia, en un repliegue especulativo sobre sí misma, una ciencia que reúne sus elementos y les da forma poética. Tanto la vuelta hacia lo micro como la reunión entre ciencia y arte parecen encontrarse habilitados, como veremos a continuación, por el momento histórico del campo epistemológico, porque como expresa con lucidez desde las filas del ensayo literario Marcelo Cohen, a partir del siglo XX "da la impresión de que la ciencia se hace más literaria" (2003, p. 143).

Una certeza que se desintegra...

Existe a partir de comienzos del siglo XX, un giro epistemológico en el campo de las ciencias 
(una revolución en términos de Kuhn) al que Carneiro atento le dedica, en su Introdução..., un apartado especial. Aquel mandato de secesión disciplinar entre las ciencias, la filosofía y el arte, que se consolidó con la aparición del paradigma mecanicista newtoniano que rigió la dinámica de la ciencia moderna comienza, por entonces, a desandarse ante la emergencia de la mecánica cuántica.

En principio, el quiebre epistemológico que supuso el paradigma de la física subatómica (Weil, 2006) relegó la observación de los fenómenos materiales (lo macroscópico) a la esfera de la ciencia moderna clásica, a la que siempre habían pertenecido; la nueva física se encargaría, en cambio, del registro subatómico discontinuo (lo microscópico). Desde entonces comienzan a convivir dos modelos de ciencia: la ciencia materialista moderna, que explica los fenómenos observables; y la cuántica, que, como modelo de explicación de lo subatómico, deberá formular otro lenguaje que se adecúe al comportamiento singular de los fenómenos no observables. En tanto la dinámica de interacción entre partículas no se corresponde (e incluso entra en manifiesta contradicción) con los parámetros de funcionamiento previstos para el mundo material, la cuántica propone principios independientes de explicación de lo subatómico. Uno de ellos (al que Carneiro decide homenajear en el título de su poemario) es el denominado «principio de incertidumbre», de Werner Heisenberg.

«Heisenberg me olha / cheio de incertezas» («Heisenberg me olha»), dice la voz poética de Carneiro; y en ese verso se encuentran cifrados no solo la referencia a la cuántica y al principio de Heisenberg que inspira las múltiples descargas líricas del poemario, sino el lugar destacado de la figura del observador. Porque lo que el principio de incertidumbre deja esbozado es que a un nivel subatómico la materia no se comporta como a nivel macroscópico: aquella sustancia mínima (que acaso nunca podamos dejar de subdividir) que compone toda materia se presenta como onda o como partícula según intervenga o no un observador, en forma de artefacto de medición. Un escándalo para la percepción empírica que históricamente se ha sustentado en la irrevocable división clásica de sujeto-objeto, el principio de incertidumbre encarna así «A certeza (que) se desintegra» («Só Verdades»), en tanto la presentación de la materia depende de la presencia del observador que ya no se considera, por ende, escindido de lo observado. ${ }^{4}$

$4 \quad$ A principios del siglo XIX, el científico inglés Thomas Young realizó lo que se llamó el experimento de "la doble rendija", a fin de exponer la doble naturaleza corpúsculo-ondular de la luz. En dicho experimento se realizan disparos no simultáneos de electrones contra un panel que posee dos rendijas. Al observar luego el patrón de impacto detrás del panel, lo que queda de manifiesto es que el electrón disparado de forma singular sale como una partícula y se convierte luego en una onda de potencialidades (se multiplica y genera un patrón de interferencia) para atravesar al mismo tiempo ambas ranuras. Todas estas posibilidades están en superposición una con la otra. Dado los desconcertantes resultados, una segunda fase del experimento contempló la instalación de un dispositivo de medición junto a la ranura, para que registrara el proceso, con lo que se esperaba determinar por cuál ranura pasaba la partícula. Pero en este segundo momento en el que se ubica un dispositivo que observa el comportamiento del electrón, este no se comporta como una onda sino como una partícula; es decir no se multiplica ni interfiere consigo misma generando una superposición al atravesar ambas rendijas, sino que se comporta como un objeto del universo material pasando solo por una. Por descabellado que suene, la partícula se comporta como una onda de potencialidades múltiples si no es observada, pero si en cambio se la observa, se muestra como una unidad sustancial del universo material, es decir, como una partícula-mónada. Por eso se habla de que la presencia del observador hace colapsar la función onda.

En este contexto, la incorporación del «principio de incertidumbre» a la mecánica cuántica operó como una resolución a este problema; aunque más que una resolución resulte un mero planteamiento del mismo, como se deja leer en el título («La interpretación de Copenhague») que se le dio al consenso alcanzado por Niels Bohr, Max Born y Heisenberg. Esto es: no es posible medir simultáneamente la trayectoria y la velocidad de una partícula porque al incluir cualquier herramienta de medición alguno de los dos valores se ve afectado. De ahí que las leyes de la física subatómica tuvieron un carácter más estadístico y probabilístico que se alejaba del determinismo de la física materialista newtoniana (De Broglie, 1952; Heisenberg, 1985). 
Ahora bien, que este nuevo paradigma científico de la física subatómica le dé un rol determinante a la figura del observador en la configuración o presentación del universo material a niveles esenciales o profundos, genera toda una serie de implicancias nada despreciables. En el registro filosófico, en primera instancia, esto supuso que los propios físicos cuánticos (Schrödinger, 1984) vieran la necesidad de revisar la filosofía idealista kantiana (a la que la ciencia moderna nunca había tenido necesidad de recurrir) en busca de fundamentos que permitieran pensar esta nueva cosmovisión en la que el sujeto evidentemente no podría entenderse de forma independiente respecto de la imagen del universo material que lo contempla. Claro está, a partir de las constataciones de la cuántica, que de ninguna manera podía seguir adjudicándosele al universo material el estatuto certero de lo externo, o cualquier tipo de autonomía respecto de las facultades innatas por medio de las cuales el sujeto, por el contrario, organiza la síntesis espacio-temporal sobre la que se apoya toda percepción del mundo, como propondría Kant.
Lo que es mucho más interesante aún, en segundo lugar, es que la centralidad que adquiere a partir de aquí el observador, no hace sino remitiros a un renovado acercamiento entre la ciencia y el arte, como señalaba Marcelo Cohen; o, mejor dicho: a una específica aproximación de la ciencia al territorio del arte. La literatura, dominio por excelencia de la percepción y registro subjetivos, reclama entonces, apelando a la legitimidad de los principios de la cuántica, nuevas validaciones para sus observaciones, como también nota con lúdica precisión el escritor César Aira:

Habrán reconocido el principio de Heisenberg, según el cual el observador, o la observación misma, modifica las condiciones objetivas del hecho. Más aún: disuelve la posibilidad de que el hecho tenga condiciones objetivas, lo vuelve observación, transformación, singularidad absoluta. El arte no debió esperar al descubrimiento de las partículas subatómicas para ver actuar el principio de Heisenberg, porque era la condición original de su funcionamiento, como lo es del funcionamiento del lenguaje: las palabras son delegados nuestros en el mundo, en la naturaleza, y allí se ocupan de cambiar los contornos de las cosas, o de darles contorno. Más en general, podría decirse que el principio de Heisenberg es la condición primera del funcionamiento de la conciencia; pero no de la inimaginable conciencia en sí, sino hecha lenguaje. (1993, p. 57).

De este modo, la ficcionalización de la ciencia subatómica (o, en este caso específico que nos atañe: su ingreso en la lógica del registro lírico) se ve facilitada por la compatibilidad de los nuevos presupuestos de la ciencia y los propios de la serie literaria. La ciencia ratifica la primacía del observador, figura históricamente soberana del dominio de la literatura, al tiempo que habilita una nueva visión holística (que pone en cuestión la escisión clásica entre sujeto-objeto) que a la literatura no puede sino resultarle atractiva. ${ }^{5}$ En este contexto, el próximo paso será entonces que el andamiaje científico funcione en la dinámica literaria para pensar sus problemas más íntimamente cercanos e incipientes, entre ellos no podría estar ausente la reflexión en torno a los usos y estatuto de la palabra.

\footnotetext{
La cosmovisión holística que inaugura la cuántica se sustenta, por un lado, como ya se ha mencionado, en la puesta en cuestión de la división materialista clásica de sujeto-objeto, a partir del "principio de incertidumbre". No obstante, el segundo principio que viene a reforzar esto es el conocido como "entrelazamiento cuántico", formulado en 1935 por Erwin Schrödinger. El "principio de entrelazamiento" es producto de la constatación de que a un nivel subatómico las descargas que se le realizan a una partícula repercuten en otras con las que esta no tiene contacto. Por eso se afirma que a niveles subatómicos toda la materia estaría "entrelazada", sería un todo. Este principio se encuentra ampliamente ficcionalizado en la narrativa de Carneiro ( $y$ en la de tantos otros autores vinculados al género) y está al servicio de la indagación sobre los fenómenos de telepatía y telequinesis (Martínez, 2018).
} 
Una ciencia subatómica de la palabra

La función de la palabra cobra, en el contexto del poemario, un valor adicional; un plus además del que ya poseía según las teorías sobre la parapsicología y la hipnosis a las que Carneiro siempre suscribió. En el texto de divulgación $O$ Mundo Misterioso do Hipnotismo (1963, pp. 3 y 134), Carneiro le asigna a la palabra (en la línea del psicoanálisis) un valor sanador o de cura de dolencias físicas y psíquicas a través de su poder esencialmente sugestivo. En su narrativa, la escritura es, además, forma de exposición de conflictos ocultos y vía de su eventual tramitación simbólica, como expondrán varios de los protagonistas de sus relatos e incluso, con frecuencia, la figura ficcionalizada del autor mismo que en estos ocasionalmente irrumpe (Martínez, 2018).

En este sentido, las teorías de la física cuántica vienen a redoblar, desde otro ángulo, este valor que Carneiro le otorga tempranamente a toda enunciación: si el observador es determinante en la configuración del universo material, entonces la intervención directa de su palabra es fundación de órdenes de sentido; es decir, presupone la construcción de mundos. En tanto la presencia del observador determina que lo real se presente como una formación de partículas determinadas que hacen co- lapsar aquella totalidad inasible de la función onda, la existencia del universo se equipara a la del poema; y la figura del poeta, observador y enunciador, a la del Dios cuya Creación es, ante todo, verbo: «O universo é um verso / o mais longo e imperfeito, / feito por um Deus alucinado / com mania de grandeza.» («Mania de Grandeza»). De modo que la vieja máxima religiosa presente en las Escrituras por la que el mundo en sus comienzos fue enunciación divina (Dios dijo: ‘¡Hágase la luz!' Y la luz se hizo), encuentra en el seno lírico de Quânticos da Incerteza explicación científica a partir del nuevo estatuto que adquiere la figura del observador.

No obstante, en tanto el poemario sigue el mismo paradigma, la situación se complejiza conforme avanza la sucesión de poemas que abordan el problema en torno a la palabra; hay, por lo tanto, una progresión reflexiva. Fundado por el observador, el poema es, al igual que el universo, «nevoeiro de átomos» («Bola de Cristal»), una niebla densa de partículas en la que lo real se presenta como materia, al tiempo que oculta todas las posibilidades abortadas ante el colapso de la función onda. Porque en mecánica cuántica la intervención del observador implica la eclosión de la partícula tanto como el eclipse de aquella forma potencial de lo real en la que la onda se expande superando cualquier po- sibilidad acotada de la partícula. El poema significa entonces la creación de un mundo que se muestra como una formación de átomos, cuya presencia material, al mismo tiempo, oculta lo que hace colapsar como posibilidad sensible en el mismo acto enunciativo de creación.

El poema como niebla densa de partículas, es organización y presentificación de lo real como materia, pero también interrupción violenta de una inasible realidad múltiple que se asemeja a la «cosa-en-sí» kantiana. Por eso, para Carneiro, el poema (al igual que cualquier formación material del universo) es además una «bola da roleta, / sorteia a porta aberta no tempo», un «espermatozoide» que llega a destino, «Sexo das palabras» («Bola da Roleta»), una «errática partícula» («Errática partícula»). Es decir, una formación aleatoria, fortuita, arbitraria, azarosa en la que cualquier alternativa puede, por combinación, resultar; al igual que sucede en la instancia en la que un espermatozoide logra su objetivo de fecundación entre millones que finalmente perecen.

Toda realidad no hace sino ocultar, irrealizadas, múltiples alternativas detrás de la elección: "Cada letra tem outros átomos, / cada palavra veste outra roupa...» («Secam por Dentro»); esto es: cada palabra (e incluso unidades más mínimas 
como las letras) podría encontrar otros destinos, mostrarse con otras vestimentas, exponer otros costados cuyas presencias se volverían igualmente legítimas por la sola proferición caprichosa de un observador que interviene, como Dios, creando un mundo de sentido. ${ }^{6}$ Tal vez por eso el poema es al mismo tiempo presentificación, eclosión como realidad material sensible, y niebla que se extiende como un velo sobre aquello que permanece en la oscura potencia de la irresolución.

El ejercicio escritural que se inicia con la observación del poeta, sin duda, marca un límite entre lo que es traído a la presencia y lo que no, como diría Heidegger en "El origen de la obra de arte" (2000 [1935]), en el proceso de desocultamiento. El poema es así un acto no despojado de violencia en el que se instaura un mundo. Mejor dicho: toda apertura de un mundo necesariamente implica la violencia de un acto enunciativo en el que ciertas palabras (como los espermatozoides que logran fecundar) advienen en la presencia de lo proferido a costa de la ausencia u ocultamiento de otras. Por eso, ya en Exemplos do Insoldavél (1985), la intervención del poeta, el avanzar incisivo de su pluma («a caneta») es para Carneiro la operación de un «bisturí ciego» («Bisturi cego»), un gesto de fundación que abre universos insospechados y azarosos, que en su autonomía luego no hacen sino escapar de los dominios del poeta:

A caneta, morna e lenta me acompanha nesta tarefa de traçar o mapa, traduzir a gota deslizando pelas veias.

$\mathrm{O}$ que fazer ao abrir a porta e encontrar um espaço de planetas?
Pero, aquellas ontologías a las que la poesía da vida como mundos autónomos no pueden ser, paradójicamente, sino medias verdades o verdades a media: «Temo que o poema se arrastre / nas meias verdades» («Neurônios Alheios») «cada palavra é nevoeiro / como dizer coisas objetivas, ponderadas e certas?» («Quânticos da Incerteza»). El terreno que abre el poema es incierto, discontinuo, intermitente, presentación que se funda sobre la interrupción de múltiples posibilidades abortadas que no obstante resuenan latentes en cada palabra. La escritura, una tirada de dados, un Big Bang a partir del cual se ordenan, aleatorios, los pedazos. El poema funda entonces un registro simbólico que muestra al tiempo que oculta, presenta, dice, expone solo un ángulo o fase. La totalidad inasible de la onda sede paso ante la ciencia incierta, subatómica de la palabra que funda embrionariamente todo orden de sentido.

$6 \quad$ Esta idea que encuentra nuevos argumentos teóricos en los principios de la mecánica cuántica ya está presente, no obstante, en el primer poemario de Carneiro, Ángulo e face (1949), al cual recorre una Stimmung melancólica que se funda en una consciencia sobre la imposibilidad de presentación de la totalidad. Precisamente, el poema sólo puede presentar fragmentariamente una perspectiva, un ángulo, una fase, un recorte observacional (lingüístico) de los fenómenos o una percepción siempre distorsionada del recuerdo. El primer verso del último poema del libro, titulado de forma homónima al poemario, dice: «Tristeza das palabras mudas/ que soam lá dentro/ e se dispersam/ no silêncio/ de inelutáveis/ amarras.» 


\section{Referencias}

Aira, C. (1993). La genealogía del monstruo. Paradoxa, (7), 55-71.

Bell, A. y Molina Gavillán, Y. (2003). Cosmos latinos: an anthology of science fiction from Latin America and Spain. Middletown, CT: Wesleyan University Press.

Carneiro, A. (1949). Ángulo e face. São Paulo: Cadernos do clube de poesia.

Carneiro, A. (1966). Espaçopleno. São Paulo: Clube de poesia.

Carneiro, A. (1963). O Mundo Misterioso do Hipnotismo. São Paulo: Edart.

Carneiro, A. (1967). Introdução ao Estudo da 'Science Fiction. São Paulo: Conselho Estadual de Cultura.

Carneiro, A. (2007). Quânticos da Incerteza. Atibaia, SP: Redijo.

Causo, R. de S. (2003). Ficção científica, fantasia e horror no Brasil-1875 a 1950. Belo Horizonte: Editora da UFMG.

Cohen, M. (2003). ¡Realmente fantástico! Y otros ensayos. Buenos Aires: Norma.

De Broglie, L. (1952). La física nueva y los cuantos. Buenos Aires: Losada.

Duarte, O. (2010a). No fim da página sou o eco: breve estudo da poesia de André Carneiro. Instrumento crítico. Revista de Estudos da Linguagem, Universidade de Rondônia (Campus de Vilhena), n. 3 , pp. 53-82.

Duarte, O. (2010b) (comp.). Exemplos do Insoldavél - Uma antología. Instrumento crítico. Revista de Estudos da Linguagem, Universidade de Rondônia (Campus de Vilhena), n. 3, pp. 178-188.

Dunbar, D. (1976). Unique motifs in Brazilian science fiction. (Tesis de Doctorado). University of Arizona, Tucson, EE. UU.

Gasparini, S. (2012). Espectros de la ciencia. Fantasías cientificas de la Argentina del siglo XIX. Buenos Aires: Santiago Arcos.

Heidegger, M. (2000 [1935]). El origen de la obra de arte. Arte y poesía (Samuel Ramos trad.). México: Fondo de Cultura Económica.

Heisenberg, W. (1985 [1955]). La imagen de la naturaleza en la fisica actual (G. Ferraté trad.). Barcelona: Orbis. 
Lundwall, S. (1986). Aventuras en la jungla de pulpa (Carlos Gardini, trad.). El Péndulo, (13), 67-78.

Martínez, L. (2018). André Carneiro: la ciencia ficción y los límites de la literatura. Estudos de Literatura Brasileira Contemporânea, (54), 211-230.

Pereira, F. da C. G. (2005). Fantástica margem: o cânone e a ficção cientifica brasileira. (Tesis de Maestría). Pontifícia Universidade Católica do Rio de Janeiro, Rio de Janeiro, Brasil.

Schrödinger, E. (1984 [1956]). Mente y materia (J. Wasensberg, trad.). Barcelona: Tusquets.

Snow, C. P. (2000). Las dos culturas. Buenos Aires: Nueva Visión.

Suvin, D. (1984). Metamorfosis de la ciencia ficción: sobre la poética y la historia de un género literario (F. Patán López trad.). México: Fondo de Cultura Económica.

Weil, S. (2006 [1966]). Sobre la ciencia (S. Mattoni, trad.). Buenos Aires: El Cuenco del Plata. 
